\title{
Photoinduced intramolecular charge separation in a polymer solid below the glass transition temperature
}

\section{$\operatorname{AUTHOR}(S)$ :}

Benten, H; Ohkita, H; Ito, S; Yamamoto, M; Tohda, Y; Tani, K

\section{CITATION:}

Benten, $\mathrm{H}$... [et al]. Photoinduced intramolecular charge separation in a polymer solid below the glass transition temperature. JOURNAL OF CHEMICAL PHYSICS 2005, 123(8): 084901.

\section{ISSUE DATE:}

2005-08-22

URL:

http://hdl.handle.net/2433/39766

\section{RIGHT:}

Copyright 2005 American Institute of Physics. This article may be downloaded for personal use only. Any other use requires prior permission of the author and the American Institute of Physics. 


\title{
Photoinduced intramolecular charge separation in a polymer solid below the glass transition temperature
}

\author{
Hiroaki Benten, Hideo Ohkita, and Shinzaburo Ito $^{\text {a) }}$ \\ Department of Polymer Chemistry, Graduate School of Engineering, Kyoto University, Katsura, Nishikyo, \\ Kyoto 615-8510, Japan \\ Masahide Yamamoto \\ Faculty of Science and Engineering, Ritsumeikan University, Kusatsu, Shiga 525-8577, Japan \\ Yasuo Tohda and Keita Tani \\ Division of Natural Science, Osaka Kyoiku University, Asahigaoka, Kashiwara, Osaka 582-8582, Japan
}

(Received 24 January 2005; accepted 30 June 2005; published online 30 August 2005)

\begin{abstract}
Photoinduced intramolecular charge separation (CS) in a polar polymer glass, cyanoethylated pullulan (CN-PUL), was studied below the glass transition temperature $\left(T_{g}=395 \mathrm{~K}\right)$. A series of three carbazole (Cz: donor)-cyclohexane (S: spacer)-acceptor (A: acceptor) molecules (Cz-S-A) was used as intramolecular donor-acceptor dyads. The photoinduced CS rate was evaluated by the fluorescence decay measurement at temperatures from 100 to $400 \mathrm{~K}$. The CS rate $\left(k_{\mathrm{CS}}\right)$ increased above $200 \mathrm{~K}$ even far below $T_{g}$ where micro-Brownian motions of the whole polymer chain are frozen. Below $200 \mathrm{~K}$, on the other hand, $k_{\mathrm{CS}}$ showed weak dependence on temperature. The temperature dependence of $k_{\mathrm{CS}}$ is discussed in terms of the dielectric relaxation time of the polymer matrix. Consequently, CS below $T_{g}$ was well explained by a thermally nonequilibrium electron transfer (ET) formula above $200 \mathrm{~K}$ and by a two-mode quantum-mechanical ET formula below 200 $\mathrm{K}$. The increase in $k_{\mathrm{CS}}$ above $200 \mathrm{~K}$ is mainly caused by a thermally activated low-frequency matrix mode originating from the side-chain relaxation of polar cyano groups. The weak temperature dependence of $k_{\mathrm{CS}}$ can be explained by a nuclear-tunneling effect caused by a high-frequency matrix mode $\left(\hbar \omega_{H}=250 \mathrm{~cm}^{-1}\right)$ and an intramolecular vibrational mode $\left(\hbar \omega_{Q}=1300 \mathrm{~cm}^{-1}\right)$. The high-frequency mode of the polymer matrix was attributed to a vibrational or librational motion of polar groups in the CN-PUL glassy solid. (C) 2005 American Institute of Physics.
\end{abstract}

[DOI: $10.1063 / 1.2008249$ ]

\section{INTRODUCTION}

Electron transfer (ET) in a polar solution is controlled by various factors: distance $r$ from a donor (D) to an acceptor (A), electronic coupling $V$ of the reactant with the product, free-energy gap of the ET reaction $-\Delta G^{o}$, reorganization energy $\lambda$, solvent polarity, dielectric relaxation time of the solvent $\tau$, and temperature $T \cdot{ }^{1-8}$ In particular, the thermal fluctuation of polar solvent molecules leads the reactant to a transition state by exchanging energy and momentum with the reactant. The ET theory developed by Marcus is based on the assumption that a thermal equilibrium is established in the solvent fluctuation during the ET event. Under the thermal equilibrium condition, the ET rate $\left(k_{\mathrm{ET}}\right)$ is determined not by the solvation dynamics, that is $\tau$, but by the static polarity of the solvent. The solvent can be treated as a dielectric continuum and its static polarity is characterized by a static dielectric constant $\varepsilon_{S}$, which dominates $k_{\mathrm{ET}}$ through $-\Delta G^{o}$ and $\lambda$. The Marcus theory well describes the various ET reactions in solutions. ${ }^{2,3,6-9}$ In general, the thermal equilibrium assumption is valid for the ET reaction in a solution where the solvent motion with a dielectric relaxation time of

\footnotetext{
a) Author to whom correspondence should be addressed. Fax: +81-753832617. Electronic mail: sito@photo.polym.kyoto-u.ac.jp
}

$\tau$ is sufficiently faster than the ET event, $\left(1 / \tau \gg k_{\mathrm{ET}}\right)$. On the other hand, the assumption breaks down if the solvent relaxation is equal to $\left(1 / \tau \sim k_{\mathrm{ET}}\right)$ or slower than $\left(1 / \tau<k_{\mathrm{ET}}\right)$ the ET event. Under these thermally nonequilibrium conditions, $k_{\mathrm{ET}}$ will be dependent on $\tau^{7,8,10-14}$ In fact, there have been several reports on the solvent-controlled ET. ${ }^{15-18}$ Heitele and co-workers studied photoinduced intramolecular charge separation (CS) in highly viscous propylene glycol solvent. ${ }^{15,16}$ They found a smooth transition of CS from nonadiabatic to solvent-controlled adiabatic behavior with an increase in the solvent relaxation time $\tau$ at lower temperatures. Recent progress in spectroscopic instruments allows one to directly observe the ultrafast ET in a time domain faster than a picosecond where the thermal equilibrium is not established yet. ${ }^{711-14,19-30}$ Some studies reported that $k_{\mathrm{ET}}$ is proportional to $1 / \tau$. ${ }^{7,13,14,19,20,24,26}$ Others reported the existence of ET faster than orientational motions of the solvent. ${ }^{7,13,14,21-25,27-30}$ The ultrafast ET, which is faster than $1 / \tau$, indicates that there must be nuclear motions faster than the orientational motions to satisfy the energy conservation law during the ET process. To explain the ultrafast ET, several researchers introduced intramolecular vibrational motions ${ }^{10,22,23,29-32}$ or fast solvent motions ${ }^{12-14,24,25,27,28,33-35}$ such as molecular vibration, inertial motion, and libration as another reaction coordinate under thermal equilibrium in ad- 
dition to the reaction coordinate of thermally nonequilibrium solvent orientational motions. Recently, fast solvent motions contributing to solvation have been revealed by subpicosecond time-dependent fluorescence Stokes shift measurements or a variety of nonlinear spectroscopic methods. ${ }^{24,36-43}$ These solvent motions interact with the reactant on a time scale faster than $0.1 \mathrm{ps}$ and they have a temperature-independent relaxation time. Some theoretical ET models introducing these fast motions predict that $k_{\mathrm{ET}}$ is mainly dominated by the fast motions rather than following the orientational motions of the solvent. ${ }^{24,28,33,34}$ Even now, however, the influence of those solvent dynamics on the ET reaction is not understood completely.

Compared with these extensive studies on ET in solutions under both thermal equilibrium and thermally nonequilibrium conditions, there have been few experimental studies on ET in polymer solids. ${ }^{44-46}$ Different from a polar Debye solvent, ${ }^{47,48}$ polymer solids have various dielectric relaxation modes and each mode has a wide distribution in $\tau:{ }^{48-52}$ motions of a long segment in the main chain, local motions of a short segment in the main chain, rotational motions of a polar group in the side chain, and damped oscillations of a polar group. Above the glass transition temperature $\left(T_{g}\right)$, the motions of the whole polymer chains are allowed and the side-chain relaxation time merges with the main chain. Our previous studies showed that photoinduced CS above $T_{g}$ can be explained by the ET formula with a quantum mode of intramolecular vibration and a classical mode of lowfrequency orientational motions of the polymer matrix under a thermal equilibrium condition. ${ }^{53,54}$ In other words, even in polymer solids, thermal fluctuations of polar groups leading to the ET reaction can be considered in a thermal equilibrium above $T_{g}$.

Below $T_{g}$, on the other hand, the motion of the main chain is frozen and dielectric relaxations are mainly due to a variety of local motions induced by the side-chain relaxation. Inhomogeneity in the relaxation time is more remarkable in the side-chain relaxation rather than the main-chain relaxation. Thus, it is difficult to specifically determine the relaxation time $\tau$ that contributes to the ET event below $T_{g}$. In polymer glass, most part of the dielectric relaxation is slower than the ET event. Providing that ET is effectively promoted by these slow motions, it would proceed under a thermally nonequilibrium condition. However, even below $T_{g}$, local fast fluctuations still remain and a high-frequency tail of dielectric relaxation extends into a time scale faster than nanoseconds. ${ }^{52,55}$ In fact, there is a temperature-independent solvation owing to inertial motions in polymer glass as in a solution. ${ }^{56-60}$ Providing that ET is effectively promoted by these fast motions, it would proceed under a thermal equilibrium condition. Owing to the wide distribution of dielectric relaxations in polymer glass, ET in polymer solids should be different from that in a polar Debye solution. However, there have been no systematic studies dealing with this problem.

Here we studied photoinduced CS in carbazole-spaceracceptor ( $\mathrm{Cz}-\mathrm{S}-\mathrm{A})$ dyad molecules embedded in a cyanoethylated pullulan (CN-PUL) polymer glassy solid. Dielectric motions in the polymer were examined at relaxation time $\tau$ with the Cole-Cole distribution function $\phi(\tau){ }^{48,50,61}$ The av- eraged CS rate $\left\langle k_{\mathrm{CS}}(\tau)\right\rangle$ was calculated from an integral of $k_{\mathrm{CS}}(\tau)$ weighted by $\phi(\tau)$ over a wide time range of $\tau$. The CS of Cz-S-A is suggested to be effectively coupled to fast dielectric motions corresponding to the lifetime of the $\mathrm{Cz}$ donor in the excited state. The effective dielectric constant $\varepsilon_{\text {eff }}$ coupled with CS can be directly evaluated by using a fluorescent polarity probe of carbazole-terephthalate cyclophane (Cz-TP). ${ }^{62}$ Consequently, the photoinduced CS in the glassy polymer solid was well explained by the thermally nonequilibrium ET formula from $T_{g}$ to $200 \mathrm{~K}$ and by the two-mode quantum-mechanical ET formula under the thermal equilibrium condition below $200 \mathrm{~K}$. The quantum-mechanical ET formula includes a high-frequency mode of the polymer matrix and a high-frequency intramolecular vibrational mode.

\section{EXPERIMENT}

\section{A. Materials}

\section{Intramolecular donor-spacer-acceptor compounds}

A series of three carbazole ( $\mathrm{Cz}$ : donor)-cyclohexane $(\mathrm{S}$ : spacer)-acceptor (A: acceptor) (Cz-S-A) molecules was synthesized and used as intramolecular D-A dyads. $N$-Ethylcarbazole $(\mathrm{EtCz})$ was synthesized and used as a reference. Details of the synthesis have been described elsewhere. $^{53}$

\section{Cz-TP fluorescent polarity probe}

$\mathrm{Cz}$-TP was synthesized by photodeselenation of the selenacarbazolophane. Details of the synthesis have been described elsewhere. ${ }^{63}$

\section{Cyanoethylated pullulan}

As a polymer matrix, cyanoethylated pullulan ${ }^{62}(\mathrm{CN}-$ PUL) was used in this study, which was kindly donated from Shin-Etsu Ltd. The glass transition temperature of CN-PUL was $T_{g}=395 \mathrm{~K}$, which was measured by differential scanning calorimetry. The refractive index of CN-PUL film was $n=1.50$ at $300 \mathrm{~K}$, which was measured by using an Abbe refractometer (Atago) with an index-matching fluid $\left(n_{\mathrm{D}}\right.$ $=1.520$ at $298 \mathrm{~K}$ ). The CN-PUL was purified by reprecipitation from an acetone solution into hexane three times before use.

Figure 1 shows the chemical structures of $\mathrm{Cz}-\mathrm{S}-\mathrm{A}, \mathrm{EtCz}$, $\mathrm{Cz}-\mathrm{TP}$, and CN-PUL polymer.

\section{B. Methods}

\section{Preparation of polymer film samples}

Polymer films of CN-PUL doped with $\mathrm{EtCz}$ or $\mathrm{Cz}-\mathrm{S}-\mathrm{A}$ were prepared by the solution casting method. The $\mathrm{EtCz}$ and $\mathrm{Cz}-\mathrm{S}-\mathrm{A}$ compounds were dissolved in an acetonitrile (Nacalai Tesque, spectroscopic grade) solution of CN-PUL so that the concentration of chromophores in the final polymer film was in the order of $10^{-3} \mathrm{~mol} \mathrm{~L}^{-1}$. The polymer solution was dropped onto a quartz plate $\left(15 \times 15 \times 1 \mathrm{~mm}^{3}\right)$, dried for several days under atmospheric pressure at room temperature, and for $12 \mathrm{~h}$ under reduced pressure above $T_{g}$. Polymer films had a thickness of about $50 \mu \mathrm{m}$. 
a) $\mathrm{Cz}-\mathrm{S}-\mathrm{A}$

b) $\mathrm{EtCz}$
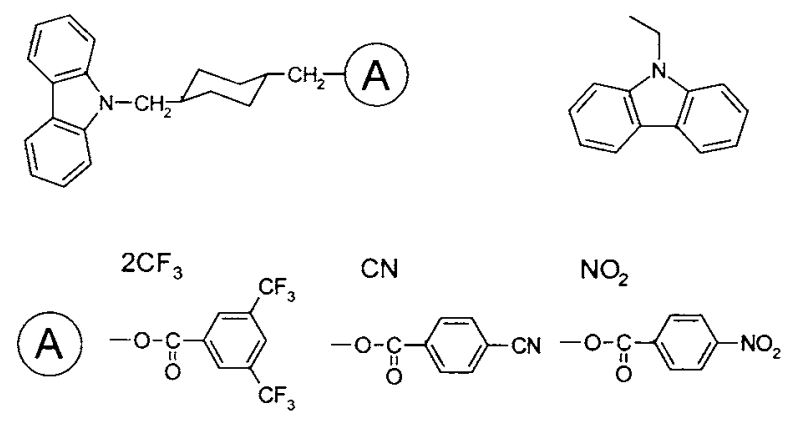

c) $\mathrm{Cz}-\mathrm{TP}$

d) CN-PUL
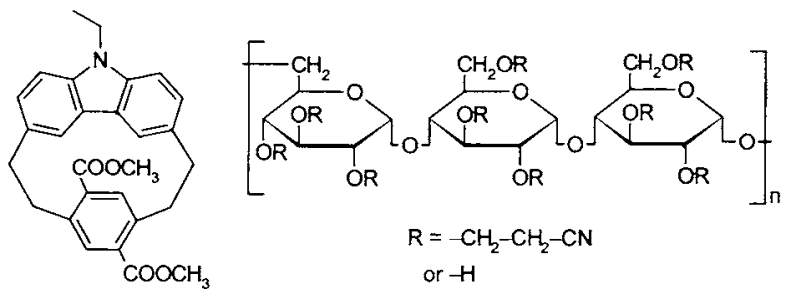

FIG. 1. Chemical structures: (a) three kinds of intramolecular Cz-S-A, (b) $N$-ethylcarbazole $(\mathrm{EtCz})$, (c) a fluorescent polarity probe carbazoleterephthalate cyclophane (Cz-TP), and (d) a polymer glassy matrix cyanoethylated pullulan (CN-PUL).

\section{Fluorescence decay measurements}

Fluorescence decay was measured by the time-correlated single-photon-counting method. The excitation light source was third-harmonic pulses $(295 \mathrm{~nm})$ generated from a modelocked Ti:sapphire laser (Spectra-Physics, Tsunami 3950) that was pumped by an $\mathrm{Ar}^{+}$laser (Spectra-Physics, BeamLok 2060). The fluorescence emission was detected with a photomultiplier (PMT; Hamamatsu Photonics, R3234) through a monochromator (Ritsu, MC-10N) with a cut-off filter (UV34) for the excitation light. The details of this apparatus have been described elsewhere. ${ }^{64}$ The total instrument response function has a full width at half maximum (FWHM) of ca. $750 \mathrm{ps}$ at the excitation wavelength. The decay data were fitted with sums of exponentials that were convoluted with the instrument response function by the nonlinear leastsquares method.

\section{Temperature control}

The temperature of film samples cast on a quartz plate was controlled in a cryostat (Iwatani Plantech Corp., CRT510) with a temperature control unit (Scientific Instruments, Model 9650).

\section{Dielectric relaxation measurements of $C N-P U L$}

Dielectric relaxation spectra were measured for the $\mathrm{CN}$ PUL film with a $L C R$ meter (Ando Electrics, Japan) at a temperature of $100-400 \mathrm{~K}$ and frequency of $0.1-100 \mathrm{kHz}$.

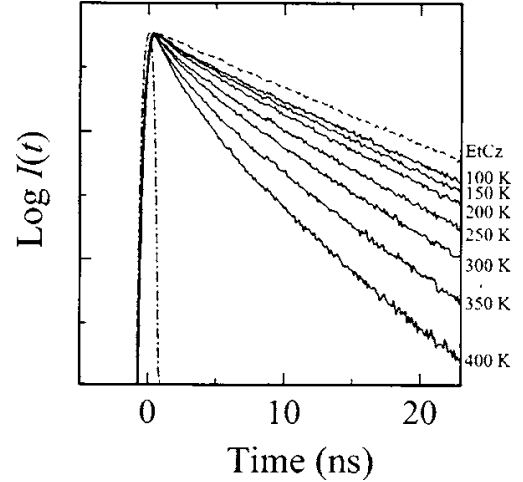

FIG. 2. Temperature dependence of fluorescence decay curve $I(t)$ for $\mathrm{EtCz}$ and $\mathrm{Cz}-\mathrm{S}-\mathrm{CN}$. The $I(t)$ of reference $\mathrm{EtCz}$ was independent of temperature from 100 to $400 \mathrm{~K}$ (a broken line). The $I(t)$ of $\mathrm{Cz}-\mathrm{S}-\mathrm{CN}$ varied with the temperature (solid lines). Seven solid lines correspond to the $I(t)$ at temperatures $100,150,200,250,300,350$, and $400 \mathrm{~K}$, from top.

\section{RESULTS AND DISCUSSION}

\section{A. Temperature dependence of $k_{\mathrm{CS}}$ of Cz-S-A in CN-PUL glassy solid}

Fluorescence decays $I(t)$ of a reference $\mathrm{EtCz}$ and $\mathrm{Cz}-\mathrm{S}-\mathrm{A}$ were measured from 100 to $400 \mathrm{~K}$. Figure 2 shows the temperature dependence of $I(t)$ for $\mathrm{EtCz}$ and $\mathrm{Cz}-\mathrm{S}-\mathrm{CN}$. The fluorescence decay of $\mathrm{EtCz}$ (broken line) was independent of temperature from 100 to $400 \mathrm{~K}$. On the other hand, the fluorescence of $\mathrm{Cz}-\mathrm{S}-\mathrm{CN}$ decayed faster at temperatures higher than $200 \mathrm{~K}$. At temperatures below $200 \mathrm{~K}, I(t)$ of $\mathrm{Cz}-\mathrm{S}-\mathrm{CN}$ was close to that of $\mathrm{EtCz}$, but even at $100 \mathrm{~K}$ there remained a clear difference in $I(t)$ between $\mathrm{Cz}-\mathrm{S}-\mathrm{CN}$ and EtCz. A similar temperature dependence of the fluorescence decay was observed for $\mathrm{Cz}-\mathrm{S}-2 \mathrm{CF}_{3}$. The fluorescence decay of $\mathrm{Cz}-\mathrm{S}$ $\mathrm{NO}_{2}$ was rapid and independent of temperature. As shown in Fig. 2, $I(t)$ did not obey a single-exponential decay over the whole temperature range measured. The multiexponential decay of $I(t)$ is probably due to a temporal distribution of the dielectric relaxation time around $\mathrm{Cz}-\mathrm{S}-\mathrm{A}$ molecules in $\mathrm{CN}-$ PUL rather than spatial inhomogeneity, because the deviation from a single-exponential decay was more remarkable at higher temperatures. All the decay curves of $\mathrm{Cz}-\mathrm{S}-\mathrm{A}$ were well fitted with the sum of three exponential functions [Eq. (1)]

$$
I(t)=I_{0} \sum_{i} A_{i} \exp \left(-t / \tau_{f i}\right) .
$$

Photoinduced CS rate $k_{\mathrm{CS}}$ was calculated by $k_{\mathrm{CS}}=1 /\left\langle\tau_{f}\right\rangle$ $-1 / \tau_{f}^{0}$, where $\tau_{f}^{0}$ is the fluorescence lifetime of EtCz and $\left\langle\tau_{f}\right\rangle$ is the averaged fluorescence lifetime of Cz-S-A. ${ }^{65,66}$

Figure 3 shows the temperature dependence of $k_{\mathrm{CS}}$ of the three $\mathrm{Cz}-\mathrm{S}-\mathrm{A}$ molecules. For $\mathrm{Cz}-\mathrm{S}-\mathrm{NO}_{2}, k_{\mathrm{CS}}$ was invariant over the whole temperatures measured from 100 to $400 \mathrm{~K}$. In contrast, $k_{\mathrm{CS}}$ of $\mathrm{Cz}-\mathrm{S}-2 \mathrm{CF}_{3}$ and $\mathrm{Cz}-\mathrm{S}-\mathrm{CN}$ increased with increasing temperature above $200 \mathrm{~K}$ while it was weakly dependent on temperatures below $200 \mathrm{~K}$. The critical temperature $T_{c}=200 \mathrm{~K}$, far below $T_{g}=395 \mathrm{~K}$, corresponds to a subtransition temperature of CN-PUL, which is ascribed to a local relaxation of side chains with polar cyano groups. ${ }^{62}$ The polar cyano groups having a large dipole moment are 


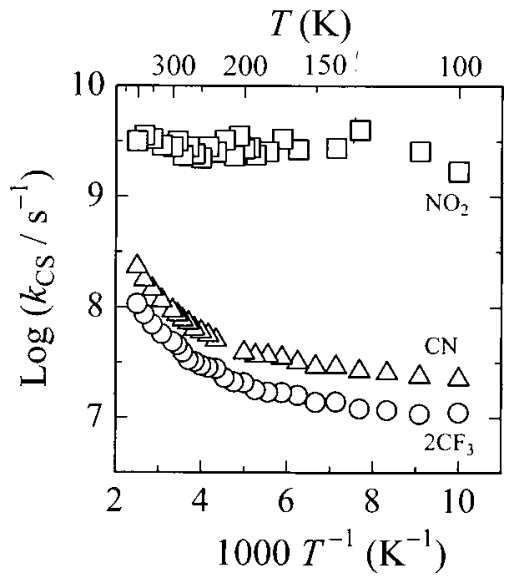

FIG. 3. Temperature dependence of $k_{\mathrm{CS}}$ from 100 to $400 \mathrm{~K}$ for $\mathrm{Cz}-\mathrm{S}-\mathrm{NO}_{2}$ (squares), $\mathrm{Cz}-\mathrm{S}-\mathrm{CN}$ (triangles), and $\mathrm{Cz}-\mathrm{S}-2 \mathrm{CF}_{3}$ (circles) in a CN-PUL glassy solid.

located at the end of the side chains where there is free volume and larger flexibility. This is why the local motions of the polar cyano groups are activated even far below $T_{g}$. Thus, the increase in $k_{\mathrm{CS}}$ of $\mathrm{Cz}-\mathrm{S}-2 \mathrm{CF}_{3}$ and $\mathrm{Cz}-\mathrm{S}-\mathrm{CN}$ above $T_{c}$ is also ascribable to the local orientational motions of the polar cyano groups in the side chains. This dielectric behavior differs from that of cyanoethylated $O$-(2-hydroxypropyl)cellulose (CN-HPC) and poly(alkyl methacrylate) where most dielectric motions are frozen below the $T_{g}{ }^{62}$ Above $T_{g}$, photoinduced CS of Cz-S-A in CNHPC and poly(alkyl methacrylate) solids could be explained by the thermal equilibrium. ${ }^{53,54}$ In CN-PUL glass, however, a wide distribution in $\tau$ should be considered to discuss CS below $T_{g}$ because there are various dielectric motions not only slower but also faster than the CS event. In Sec. III B, the temperature dependence of $k_{\mathrm{CS}}$ will be discussed in detail considering the wide distribution in the relaxation time of the CN-PUL glassy solid.

\section{B. Analysis of $\boldsymbol{k}_{\mathrm{CS}}$ based on dielectric relaxation dynamics of CN-PUL glassy solid}

The rate of nonadiabatic CS is expressed by Eqs. (2) and (3). ${ }^{2,67}$ Here, a static dielectric constant $\varepsilon_{S}$ of the solvent matrix represents thermal fluctuation of polar solvent molecules. Considering the contribution of a quantum effect involving a high-frequency intramolecular vibration to the CS reaction, $k_{\mathrm{CS}}$ is expressed by a sum of nonadiabatic CS rates $k_{\mathrm{NA}}^{0 \rightarrow n}$ from the reactant in the ground vibrational state to the product in the $n$th excited vibrational state,

$$
\begin{aligned}
& k_{\mathrm{CS}}^{\mathrm{NA}}= \sum_{n} k_{\mathrm{NA}}^{0 \rightarrow n}, \\
& k_{\mathrm{NA}}^{0 \rightarrow n}= \frac{2 \pi}{\hbar} V^{2} \frac{1}{\sqrt{4 \pi \lambda_{\mathrm{S}} k_{\mathrm{B}} T}} \frac{S^{n} \exp (-S)}{n !} \\
& \quad \times \exp \left[-\frac{\left(\Delta G_{\mathrm{CS}}+\lambda_{\mathrm{S}}+n \hbar \omega_{Q}\right)^{2}}{4 \lambda_{\mathrm{S}} k_{\mathrm{B}} T}\right], \\
& S=\frac{\lambda_{Q}}{\hbar \omega_{Q}},
\end{aligned}
$$

$$
\begin{aligned}
&-\Delta G_{\mathrm{CS}}= E\left(S_{1}\right)-\left(E_{\mathrm{ox}}^{\mathrm{D}}-E_{\mathrm{red}}^{\mathrm{A}}\right)+\frac{e^{2}}{4 \pi \varepsilon_{0}}\left\{\frac{1}{\varepsilon_{\mathrm{S}} r_{\mathrm{DA}}}\right. \\
&\left.+\frac{1}{2}\left(\frac{1}{r_{\mathrm{D}}}+\frac{1}{r_{\mathrm{A}}}\right)\left(\frac{1}{37.5}-\frac{1}{\varepsilon_{\mathrm{S}}}\right)\right\}, \\
& \lambda_{\mathrm{S}}=\frac{e^{2}}{4 \pi \varepsilon_{0}}\left(\frac{1}{2 r_{\mathrm{D}}}+\frac{1}{2 r_{\mathrm{A}}}-\frac{1}{r_{\mathrm{DA}}}\right)\left(\frac{1}{\varepsilon_{\infty}}-\frac{1}{\varepsilon_{\mathrm{S}}}\right),
\end{aligned}
$$

where $V$ is the electronic coupling matrix element, and $S$ is the electron vibrational coupling strength given as Eq. (4) by using both reorganization energy for intramolecular vibration $\lambda_{Q}$ and its vibrational quantum $\hbar \omega_{Q}$. The free-energy gap for the photoinduced CS $-\Delta G_{\mathrm{CS}}$ and reorganization energy for solvent $\lambda_{S}$ are given by Eqs. (5) (Refs. 68 and 69) and (6), respectively, where $E\left(S_{1}\right)$ is the energy level of the lowest excited singlet state of $\mathrm{EtCz}, E_{\mathrm{ox}}^{\mathrm{D}}$ and $E_{\mathrm{red}}^{\mathrm{A}}$ oxidation potential of donor $(\mathrm{Cz})$ and reduction potential of acceptor (A), respectively, $e$ the elementary charge, $\varepsilon_{0}$ the vacuum permittivity, $\varepsilon_{\mathrm{S}}$ static dielectric constant of the matrix, $r_{\mathrm{DA}}$ separation distance between the donor and acceptor, $r_{\mathrm{D}}$ and $r_{\mathrm{A}}$ spherical radii of donor and acceptor moieties, respectively, ${ }^{5.3}$ and $\varepsilon_{\infty}$ optical dielectric constant of the matrix which is equal to the squire of the refractive index of the matrix $n^{2}$,

$$
\begin{aligned}
& k_{\mathrm{CS}}(\tau)=\sum_{n} \frac{1}{1+\kappa^{0 \rightarrow n}(\tau)} k_{\mathrm{NA}}^{0 \rightarrow n}, \\
& \kappa^{0 \rightarrow n}(\tau)=\frac{4 \pi \tau}{\hbar \lambda_{\mathrm{S}}} V^{2} \frac{S^{n} \exp (-S)}{n !} .
\end{aligned}
$$

On the other hand, the CS rate is modified by Eqs. (7) and (8) under the condition that relaxations of polar solvent molecules are as slow as the CS reaction and thermal equilibrium is not established. The CS rate is a function of solvent relaxation time $\tau$, because the thermal equilibrium condition is no longer valid for the CS reaction. In other words, dynamical solvation dominates the CS rate. These equations were developed by Jortner and co-workers to introduce the dynamical dielectric property of solvent to the ET reaction. ${ }^{31,32,70,71}$ Here, $\kappa^{0 \rightarrow n}(\tau)$ is an effective adiabaticity parameter between the ground vibrational state of the reactant and the $n$th excited vibrational state of the product. Thus, solvent relaxation dynamics influence the CS rate through $\kappa^{0 \rightarrow n}(\tau)$. In normal organic liquids, the parameter can be negligible for our Cz-S-A because $\tau$ is in the order of $10^{-12} \mathrm{~s}$ and $V$ is small enough $\left(<10 \mathrm{~cm}^{-1}\right)$, that is, nonadiabatic limit $\left[\kappa^{0 \rightarrow n}(\tau) \ll 1\right] .^{53}$ In polymer glassy solids, however, the nonadiabatic limit may not be assumed because $\tau$ is generally longer than that in normal organic liquids.

Dielectric relaxation spectra of the CN-PUL glassy solid were analyzed with a single Cole-Cole function [Eq. (9) $],{ }^{48,50,61}$

$$
\varepsilon^{*}(\omega)=\varepsilon_{\infty}+\frac{\varepsilon_{\mathrm{S}}-\varepsilon_{\infty}}{1+\left(i \omega \tau_{m}\right)^{\beta}},
$$

where $\varepsilon^{*}(\omega)$ is the complex dielectric permittivity, $\tau_{m}$ the average dipolar relaxation time, $\beta(0<\beta \leqslant 1)$ a parameter describing the distribution of relaxation time, and $i$ the imagi- 


\section{$T(\mathrm{~K})$}

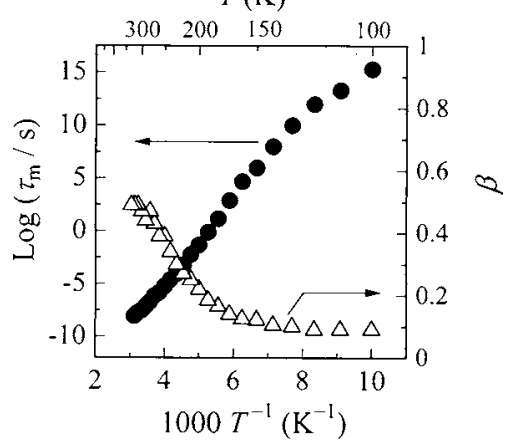

FIG. 4. Temperature dependence of the average dipolar relaxation time $\tau_{n}$ (closed circles), and a parameter $\beta$ describing the distribution of relaxation time (triangles). These are obtained by analyzing dielectric relaxation spectra of the CN-PUL with a single Cole-Cole function [Eq. (9)].

nary unit. Figure 4 shows the temperature dependence of $\tau_{m}$ (closed circles) and $\beta$ (triangles). First, $k_{\mathrm{CS}}$ of $\mathrm{Cz}-\mathrm{S}-2 \mathrm{CF}_{3}$ was calculated by the ET formula considering the thermally nonequilibrium effect [Eq. (7)] with the average dipolar relaxation time $\tau_{m}$ because of the wide distribution in relaxation time. This approximation by a single relaxation time is generally used for a polar Debye solvent. In the calculation, parameters of $V, \lambda_{Q}$, and $\hbar \omega_{Q}$ were used as follows: $V$ $=3.5 \mathrm{~cm}^{-1}, \lambda_{Q}=0.56 \mathrm{eV}$, and $\hbar \omega_{Q}=1300 \mathrm{~cm}^{-1} .53,54$ The static dielectric constant $\varepsilon_{\mathrm{S}}$ in Eqs. (5) and (6) was evaluated by the dielectric analysis with the Cole-Cole function. The optical dielectric constant $\varepsilon_{\infty}$ was calculated as the square of the refractive index $n^{2}$ of CN-PUL $(n=1.50)$ and assumed to be constant over the whole temperature range. As shown in Fig. 5 (dashed-dotted line), $k_{\mathrm{CS}}\left(\tau_{m}\right)$ fell off with decreasing temperature and considerably deviated from the experimental result. The large deviation is clearly due to the disregard of a variety of relaxation times except for $\tau_{m}$ in the CN-PUL glassy solid.

Next, the averaged CS rate $\left\langle k_{\mathrm{CS}}(\tau)\right\rangle$ was calculated from the integral of $k_{\mathrm{CS}}(\tau)$ weighted by the distribution function

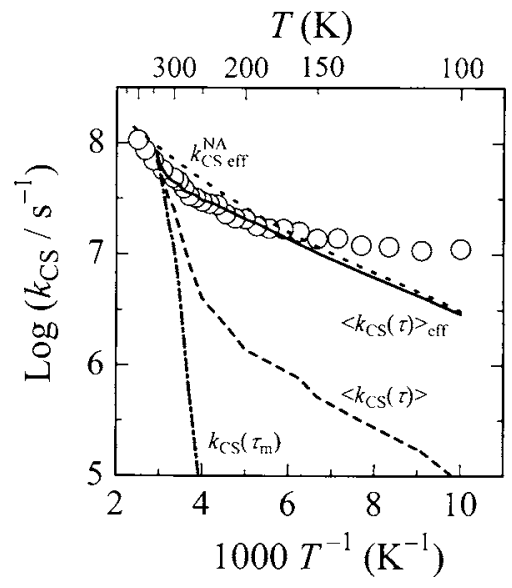

FIG. 5. Temperature dependence of the CS rate calculated by each ET equation. The dashed-dotted line represents $k_{\mathrm{CS}}\left(\tau_{m}\right)$ [Eq. (7)], the broken line $\left\langle k_{\mathrm{CS}}(\tau)\right\rangle$ [Eq. (10)], the solid line $\left\langle k_{\mathrm{CS}}(\tau)\right\rangle_{\mathrm{eff}}$ [Eq. (12)], the dotted line $k_{\mathrm{CS} \text { eff }}^{\mathrm{NA}}[\mathrm{Eq} .(2)]$, and circles are the experimental $\mathrm{CS}$ rate of $\mathrm{Cz}-\mathrm{S}-2 \mathrm{CF}_{3}$. Fitting parameters in each equation were $V=3.5 \mathrm{~cm}^{-1}, \lambda_{Q}=0.56 \mathrm{eV}$, and $\hbar \omega_{Q}=1300 \mathrm{~cm}^{-1}$. Both $-\Delta G_{\mathrm{CS}}$ and $\lambda_{\mathrm{S}}$ were evaluated with $\varepsilon_{\mathrm{S}}$ for $k_{\mathrm{CS}}\left(\tau_{m}\right)$ and $\left\langle k_{\mathrm{CS}}(\tau)\right\rangle$ and with $\varepsilon_{\text {eff }}$ for $\left\langle k_{\mathrm{CS}}(\tau)\right\rangle_{\text {eff }}$ and $k_{\mathrm{CS} \text { eff }}^{\mathrm{NA}}$, respectively. $\phi(\tau)$ over $\tau$ [Eq. (10)] to discuss the effect of the wide distribution in dielectric relaxation time $\tau$ on the CS rate. In a Cole-Cole formula, $\phi(\ln \tau)$ is expressed by Eq. (11). ${ }^{48,50,61}$

$$
\begin{aligned}
& \left\langle k_{\mathrm{CS}}(\tau)\right\rangle=\int_{0}^{\infty} k_{\mathrm{CS}}(\tau) \phi(\tau) d \tau, \\
& \phi(\ln \tau)=\frac{1}{2 \pi} \frac{\sin \beta \pi}{\left\{\cosh \left[\beta \ln \left(\tau / \tau_{m}\right)\right]+\cos \beta \pi\right\}} .
\end{aligned}
$$

Compared with $k_{\mathrm{CS}}\left(\tau_{m}\right)$, theoretical prediction of $\left\langle k_{\mathrm{CS}}(\tau)\right\rangle$ (broken line in Fig. 5) demonstrated a marked increase in CS rate above $200 \mathrm{~K}$ and a weak temperature dependence below $200 \mathrm{~K}$, although $\left\langle k_{\mathrm{CS}}(\tau)\right\rangle$ is still much smaller than the experimental result. This improvement suggests that CS in the CN-PUL glassy solid is coupled not to a representative motion with a relaxation time of $\tau_{m}$ but to a variety of motions. The remaining deviation of $\left\langle k_{\mathrm{CS}}(\tau)\right\rangle$ is probably due to slow relaxation motions, which should be considered to be frozen during the lifetime of the excited initial state. This time scale is comparable to the lifetime of $\mathrm{EtCz}$ in the excited state. Thus, dielectric dynamics with a relaxation time faster than the lifetime of $\mathrm{EtCz}$ in the excited state (13 ns) have to be evaluated.

To discuss CS in CN-PUL below $T_{g}$ on the basis of the effective dielectric dynamics with a relaxation time faster than the lifetime of $\mathrm{EtCz}$ in the excited state, we need an effective dielectric constant $\varepsilon_{\text {eff }}$ and effective distribution function $\phi(\tau)_{\text {eff }}$ corresponding to the CS reaction. As for $\varepsilon_{\text {eff }}$, it was previously demonstrated that the fluorescence polarity probe method using the carbazole-terephthalate cyclophane (Cz-TP) is a powerful tool for evaluating $\varepsilon_{\text {eff }}$ reflecting dielectric motions in a time domain shorter than the lifetime of $\mathrm{EtCz}$ in the excited state. ${ }^{62}$ Therefore, $\varepsilon_{\text {eff }}$ of the CN-PUL glassy solid was evaluated with the Cz-TP probe whose lifetime in the excited state is $35 \mathrm{~ns} .{ }^{72}$ As for $\phi(\tau)_{\text {eff }}$, the distribution function $\phi(\tau)$ should be modified according to the time window of the initial excited state, because not all dielectric motions can contribute to CS in Cz-S-A. The dielectric motions contributing to CS should be limited to those with a relaxation time $\tau$ shorter than the lifetime of $\mathrm{EtCz}$ in the excited state. Thus, we introduced a modified distribution function $\phi(\tau) I(\tau)$ as $\phi(\tau)_{\text {eff }}$. Here, the fluorescence decay $I(\tau)$ of EtCz represents the population of $\mathrm{EtCz}$ in the excited state at a time $\tau$. Figure 6(a) shows the distribution function of the side-chain relaxation time $\phi(\tau)$ of CN-PUL (solid line) at each temperature and the fluorescence decay of $\operatorname{EtCz} I(\tau)$ (broken line). As shown in the figure, most part of the $\phi(\tau)$ at each temperature is distributed in a time domain longer than that represented by the broken line $I(\tau)$, that is, the time window of the lifetime of $\mathrm{EtCz}$ in the excited state. This is why $\left\langle k_{\mathrm{CS}}(\tau)\right\rangle$ deviates from the experimental results. On the other hand, as shown in Fig. 6(b), the time domain of the effective distribution function $\phi(\tau) I(\tau)$ is consistent with the time domain of $I(\tau)$. Thus, the CS rate was evaluated by Eq. (12) with $-\Delta G_{\mathrm{CS}}\left(\varepsilon_{\mathrm{eff}}\right)$ and $\lambda_{S}\left(\varepsilon_{\mathrm{eff}}\right)$. 


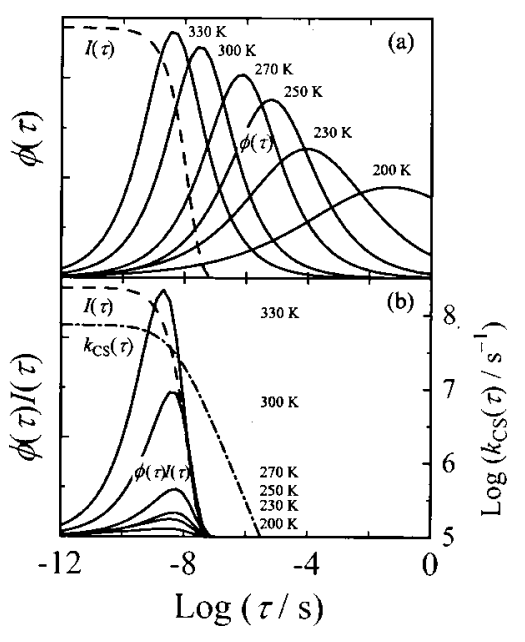

FIG. 6. Temperature dependence of dielectric distribution function from 200 to $330 \mathrm{~K}$ : (a) the distribution function of the side-chain relaxation time in CN-PUL $\phi(\tau)$ (solid lines) calculated by Eq. (11), and (b) the effective distribution function $\phi(\tau) I(\tau)$ (solid lines) obtained by multiplying $\phi(\tau)$ by the fluorescence decay $I(\tau)$ of $\mathrm{EtCz}$ (broken line). The dashed-dotted line represents $k_{\mathrm{CS}}(\tau)$ of $\mathrm{Cz}-\mathrm{S}-2 \mathrm{CF}_{3}$ at $300 \mathrm{~K}$ calculated by Eq. (7).

$$
\left\langle k_{\mathrm{CS}}(\tau)\right\rangle_{\mathrm{eff}}=\frac{\int_{0}^{\infty} k_{\mathrm{CS}}(\tau) \phi(\tau) I(\tau) d \tau}{\int_{0}^{\infty} \phi(\tau) I(\tau) d \tau} .
$$

As shown in Fig. 5 (solid line), $\left\langle k_{\mathrm{CS}}(\tau)\right\rangle_{\mathrm{eff}}$ was in good agreement with the experimental CS rate from $200 \mathrm{~K}$ to $T_{g}$. The nonadiabatic CS rate $k_{\mathrm{CS} \text { eff }}^{\mathrm{NA}}$ was also calculated by Eq. (2) with $-\Delta G_{\mathrm{CS}}\left(\varepsilon_{\text {eff }}\right)$ and $\lambda_{S}\left(\varepsilon_{\text {eff }}\right)$. In the temperature range from $200 \mathrm{~K}$ to $T_{g}, k_{\mathrm{CS} \text { eff }}^{\mathrm{NA}}$ decreased linearly with temperature whereas the experimental CS rate shows a nonlinear dependence on temperature. The deviation of $k_{\mathrm{CS} \text { eff }}^{\mathrm{NA}}$ from the experimental CS rate and the well reproduction of that by thermally nonequilibrium $\left\langle k_{\mathrm{CS}}(\tau)\right\rangle_{\mathrm{eff}}$ indicates that CS in Cz-S$2 \mathrm{CF}_{3}$ proceeds under thermally nonequilibrium condition from $200 \mathrm{~K}$ to $T_{g}$. In other words, dielectric relaxation time influences the CS rate.

For the discussion on the contribution of the effective dielectric motions to thermally nonequilibrium CS in more detail, the relaxation time $\tau$ dependence of CS rate $k_{\mathrm{CS}}(\tau)$ of $\mathrm{Cz}-\mathrm{S}-2 \mathrm{CF}_{3}$ was calculated by using Eq. (7) at $300 \mathrm{~K}$ [dashed-dotted line in Fig. 6(b)]. The $\tau$ dependence of $k_{\mathrm{CS}}(\tau)$ shows that dielectric motions with a relaxation time $\tau$ $<10^{-9} \mathrm{~s}$ cause a nonadiabatic CS and $k_{\mathrm{CS}}(\tau)$ is independent of $\tau$ while those with a relaxation time $\tau>10^{-9} \mathrm{~s}$ cause a thermally nonequilibrium $\mathrm{CS}$ and $k_{\mathrm{CS}}(\tau)$ depends on $\tau$. Therefore, within the time domain of $\phi(\tau)_{\text {eff }}$, dielectric motions contributing to thermally nonequilibrium CS are limited to those with a relaxation time $\tau>10^{-9} \mathrm{~s}$ above $200 \mathrm{~K}$. On the other hand, there still remains disagreement below $200 \mathrm{~K}$. At lower temperatures, the CS rate shows a weak temperature dependence while $\left\langle k_{\mathrm{CS}}(\tau)\right\rangle_{\mathrm{eff}}$ and $k_{\mathrm{CS}}^{\mathrm{NA}}$ eff predict a monotonous decrease with decreasing temperature. Another explanation should be considered for the weak temperature dependence of the CS rate below $200 \mathrm{~K}$.

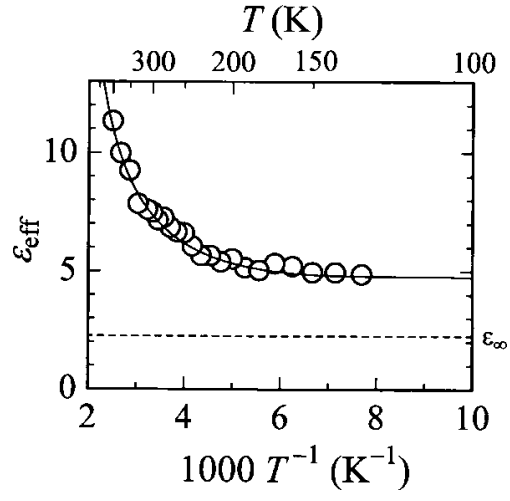

FIG. 7. Temperature dependence of $\varepsilon_{\text {eff }}$ of the CN-PUL glassy solid evaluated with a fluorescent $\mathrm{Cz}$-TP probe (circles). The solid line was drawn to reproduce experimental data of $\varepsilon_{\text {eff }}$. The broken line is optical dielectric constant $\left(\varepsilon_{\infty}=2.25\right)$.

\section{Weak temperature dependence of $k_{\mathrm{CS}}$ below $200 \mathrm{~K}$}

There are two typical explanations for the temperatureinvariant CS from a theoretical viewpoint.

(1) Activationless CS in the top region of the Marcus relation where reorganization energy is equal to the freeenergy gap for CS, $\lambda=-\Delta G_{\mathrm{CS}}$.

(2) Nuclear-tunneling effects involving a high-frequency motion of solute and/or solvent, whose vibrational quantum $\hbar \omega$ is sufficiently larger than the thermal energy $\left.k_{\mathrm{B}} T\left(\hbar \omega \gg k_{\mathrm{B}} T\right)\right)^{2,5,7,67}$

The temperature invariant $\mathrm{CS}$ rate of $\mathrm{Cz}-\mathrm{S}-\mathrm{NO}_{2}$ can be explained as in case 1: $-\Delta G_{\mathrm{CS}}=\lambda\left(\lambda=\lambda_{\mathrm{S}}+\lambda_{V}\right)$. As the effective dielectric constant $\varepsilon_{\text {eff }}=7.15$ at $295 \mathrm{~K},-\Delta G_{\mathrm{CS}}$ of Cz-S$\mathrm{NO}_{2}$ and $\lambda_{\mathrm{S}}$ were evaluated to be 1.28 and $0.72 \mathrm{eV}$, respectively. Because $\lambda_{V}=0.56 \mathrm{eV}$ as previously reported, ${ }^{53,54}$ $-\Delta G_{\mathrm{CS}}$ is equal to $\lambda=\lambda_{\mathrm{S}}+\lambda_{V} \sim 1.28 \mathrm{eV}$. This indicates that $\mathrm{CS}$ in $\mathrm{Cz}-\mathrm{S}-\mathrm{NO}_{2}$ proceeds in the top region of the Marcus relation requiring neither thermal fluctuation nor activation energy. This relation of $-\Delta G_{\mathrm{CS}} \sim \lambda$ at $295 \mathrm{~K}$ is maintained at any other temperatures including $295 \mathrm{~K}$, because $-\Delta G_{\mathrm{CS}}\left(\varepsilon_{\mathrm{eff}}\right)+\lambda_{S}\left(\varepsilon_{\mathrm{eff}}\right)$ is independent of temperature whereas each $-\Delta G_{\mathrm{CS}}\left(\varepsilon_{\text {eff }}\right)$ and $\lambda_{S}\left(\varepsilon_{\text {eff }}\right)$ depends on temperature according to Eqs. (5) and (6). ${ }^{44}$ Furthermore, the FranckCondon excited state of Cz-S-A was similar to the equilibrium one as indicated by the small fluorescence Stokes shift of EtCz. ${ }^{53}$ Consequently, it can safely be said that solvent relaxation dynamics influence little the process of CS in $\mathrm{Cz}-\mathrm{S}-\mathrm{NO}_{2}$. Thus, the CS rate of $\mathrm{Cz}-\mathrm{S}-\mathrm{NO}_{2}$ is independent of temperature. On the other hand, $\mathrm{CS}$ in $\mathrm{Cz}-\mathrm{S}-\mathrm{CN}$ and $\mathrm{Cz}-\mathrm{S}$ $2 \mathrm{CF}_{3}$ proceeds in the normal region of the Marcus relation where thermal fluctuations of the medium are required to cross the activation barrier. ${ }^{53,54}$ Thermal fluctuations of polar groups in the polymer matrix coupled with CS is represented by the effective dielectric constant $\varepsilon_{\text {eff }}$.

Figure 7 shows the temperature dependence of $\varepsilon_{\text {eff }}$ of the CN-PUL glassy solid. Even below $T_{g}, \varepsilon_{\text {eff }}$ was still larger $(\sim 5)$ than the optical dielectric constant $\left(\varepsilon_{\infty} \sim 2\right)$, which indicates the presence of dielectric motions faster than $10^{-8} \mathrm{~s}$. The temperature dependence of $\varepsilon_{\text {eff }}$ was in good agreement with the increase in the experimental $\mathrm{CS}$ rate of $\mathrm{Cz}-\mathrm{S}-2 \mathrm{CF}_{3}$ 


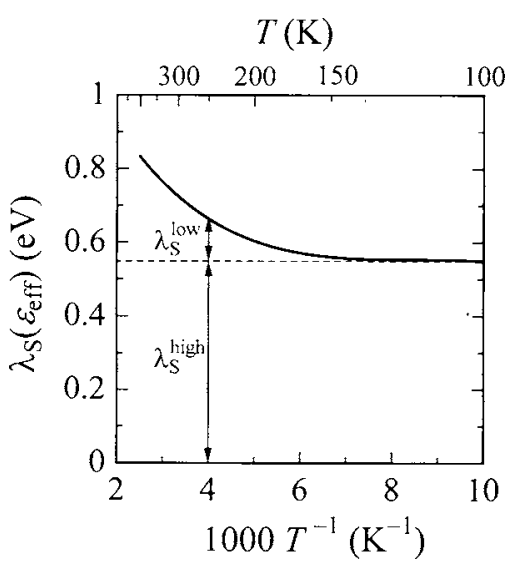

FIG. 8. Temperature dependence of effective reorganization energy $\lambda_{\mathrm{S}}\left(\varepsilon_{\text {eff }}\right)$ of the CN-PUL glassy solid, which was evaluated with $\varepsilon_{\text {eff }}$ by Eq. (6). Total effective reorganization energy $\lambda_{\mathrm{S}}\left(\varepsilon_{\text {eff }}\right)$ was divided into two components $\lambda_{\mathrm{S}}^{\text {low }}$ and $\lambda_{\mathrm{S}}^{\text {high }}: \lambda_{\mathrm{S}}^{\text {high }}$ corresponds to the temperature-invariant part of $\lambda_{\mathrm{S}}\left(\varepsilon_{\text {eff }}\right)$, $\lambda_{\mathrm{S}}^{\text {high }}=0.54 \mathrm{eV}$, and $\lambda_{\mathrm{S}}^{\text {low }}$ corresponds to temperature-dependent part of $\lambda_{\mathrm{S}}\left(\varepsilon_{\text {eff }}\right), \lambda_{\mathrm{S}}^{\text {low }}=\lambda_{\mathrm{S}}\left(\varepsilon_{\text {eff }}\right)-\lambda_{\mathrm{S}}^{\text {high }}$.

and $\mathrm{Cz}-\mathrm{S}-\mathrm{CN}$ above $200 \mathrm{~K}$ and weak temperature dependence below $200 \mathrm{~K}$. These results clearly show that CS in $\mathrm{Cz}-\mathrm{S}-\mathrm{A}$ was effectively promoted by the dielectric relaxation with $\varepsilon_{\text {eff }}$ even below $T_{g}$. The $\varepsilon_{\text {eff }}$ of poly(butyl methacrylate) solids below $T_{g}$ was as small as the optical dielectric constant. ${ }^{54}$ This difference results from the polarity and flexibility of the side chain. Orientational motions of the polar groups in poly(alkyl methacrylate) solids are highly restricted below $T_{g}$ because the polar ester group is bound at the root of the main chain. ${ }^{62}$ On the other hand, polar cyano groups of CN-PUL are highly mobile even below $T_{g}$, because they are bound at the end of the side chain. ${ }^{62^{\circ}}$ As a result, there exist dielectric relaxations of the polar group even in the CN-PUL glassy solid, which are fast enough to effectively promote the photoinduced CS.

The effective reorganization energy $\lambda_{S}\left(\varepsilon_{\text {eff }}\right)$ of the $\mathrm{CN}$ PUL glassy solid can be estimated by $\varepsilon_{\text {eff }}$ (Fig. 8). The $\lambda_{S}\left(\varepsilon_{\text {eff }}\right)$ also increased from $200 \mathrm{~K}$ and remained almost constant below $200 \mathrm{~K}$. The former part above $200 \mathrm{~K}$ was attributed to the reorganization due to low-frequency orientational motions of cyano groups $\left(\lambda_{\mathrm{S}}^{\text {low }}\right)$ and the latter part below 200 $\mathrm{K}$ was attributed to that due to high-frequency vibrational or librational motions of the CN-PUL glassy solid $\left(\lambda_{S}^{\text {high }}\right)$, respectively. Recent studies on solvation around probe molecules in solutions revealed the existence of fast motions in the time region shorter than the orientational motions. ${ }^{24,36-43}$ These fast motions are ascribed to inertial motions due to vibrational or librational motions of the solvent, which interact with the reactant on a time scale from ca. $10^{1}$ to $10^{3} \mathrm{fs}$ and the relaxation time is temperature independent. Even in polymer glassy solids, similar solvation dynamics due to libration has been observed in the same time domain. ${ }^{55-60}$ Thus, the CS reaction in Cz-S-A is driven by both the lowand high-frequency motions at temperatures above $200 \mathrm{~K}$, and mainly by the high-frequency motion remaining at temperatures below $200 \mathrm{~K}$. Providing that the fast motions are coupled with the CS reaction, nuclear-tunneling effects would be expected at low temperatures because of their large $\hbar \omega$, which causes the weak temperature dependence of the CS rate. ${ }^{2,5,7,67}$

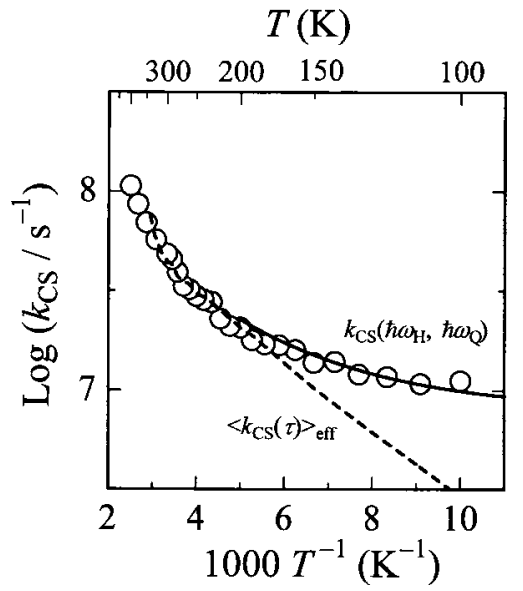

FIG. 9. Temperature dependence of $k_{\mathrm{CS}}$ of $\mathrm{Cz}-\mathrm{S}-2 \mathrm{CF}_{3}$ in the CN-PUL glassy solid. The solid line is the theoretical prediction $k_{\mathrm{CS}}\left(\hbar \omega_{H}, \hbar \omega_{Q}\right)$ calculated by Eq. (13) with $-\Delta G_{\mathrm{CS}}\left(\varepsilon_{\text {eff }}\right)$ and $\lambda_{\mathrm{S}}\left(\varepsilon_{\text {eff }}\right), \lambda_{Q}=0.56 \mathrm{eV}, \hbar \omega_{H}=250 \mathrm{~cm}^{-1}$, $\hbar \omega_{Q}=1300 \mathrm{~cm}^{-1}$, and $V=3.5 \mathrm{~cm}^{-1}$. The broken line is the theoretical prediction $\left\langle k_{\mathrm{CS}}(\tau)\right\rangle_{\text {eff }}$ calculated by Eq. (12) with $-\Delta G_{\mathrm{CS}}\left(\varepsilon_{\mathrm{eff}}\right)$ and $\lambda_{\mathrm{S}}\left(\varepsilon_{\mathrm{eff}}\right), \lambda_{\mathrm{Q}}$ $=0.56 \mathrm{eV}, \hbar \omega_{Q}=1300 \mathrm{~cm}^{-1}, V=3.5 \mathrm{~cm}^{-1}$.

To discuss the contribution of the high-frequency motions to the CS rate at low temperatures, the $\mathrm{CS}$ rate of $\mathrm{Cz}-\mathrm{S}$ $2 \mathrm{CF}_{3}$ was quantitatively evaluated by using a two-mode quantum-mechanical ET formula [Eq. (13)], ${ }^{67}$

$$
\begin{aligned}
k_{\mathrm{CS}}\left(\hbar \omega_{H}, \hbar \omega_{Q}\right)= & \frac{2 \pi}{\hbar^{2} \omega_{H}} V^{2} \exp \left[-S_{H}\left(2 \bar{n}_{H}+1\right)-S_{Q}\left(2 \bar{n}_{Q}+1\right)\right] \\
& \times \sum_{m=-\infty}^{\infty}\left(\frac{\bar{n}_{H}+1}{\bar{n}_{H}}\right)^{p(m) / 2} \\
& \times I_{|p(m)|}\left\{2 S_{H} \sqrt{\bar{n}_{H}\left(\bar{n}_{H}+1\right)}\right\} \\
& \times\left(\frac{\bar{n}_{Q}+1}{\bar{n}_{Q}}\right)^{m / 2} I_{|m|}\left\{2 S_{Q} \sqrt{\bar{n}_{Q}\left(\bar{n}_{Q}+1\right)}\right\},
\end{aligned}
$$

where

$$
\begin{aligned}
& \bar{n}_{i}=\left[\exp \left(\frac{\hbar \omega_{i}}{k_{\mathrm{B}} T}\right)-1\right]^{-1}, \quad S_{i}=\frac{\lambda_{i}}{\hbar \omega_{i}} \quad(i=H, Q), \\
& p(m)=-\frac{\Delta G_{\mathrm{CS}}+m \hbar \omega_{Q}}{\hbar \omega_{H}} .
\end{aligned}
$$

Here, $p(m)$ is taken as an integer closest to the value of $p(m)$. At temperatures below $200 \mathrm{~K}$, there exist a high-frequency matrix mode $(i=H)$ and an intramolecular vibrational mode of $\mathrm{Cz}-\mathrm{S}-\mathrm{A}(i=Q)$ because the low-frequency matrix mode is frozen. The matrix mode $(i=H)$ is ascribed to fast motions with a high vibrational quantum $\hbar \omega_{H}$ and the corresponding reorganization energy $\lambda_{H}$ is equal to $\lambda_{\mathrm{S}}^{\text {high }}$ (see Fig. 8). The intramolecular vibration is the highest-frequency quantum mode $(i=Q)$ with a vibrational quantum $\hbar \omega_{Q}$, which contributes to the reorganization energy $\lambda_{Q}$ of Cz-S-A. Using $\varepsilon_{\text {eff, }}$ $-\Delta G_{\mathrm{CS}}$ and $\lambda_{\mathrm{S}}^{\text {high }}$ were evaluated by Eq. (5) and as $\lambda_{\mathrm{S}}\left(\varepsilon_{\mathrm{eff}}\right)$ $=0.54 \mathrm{eV}$ at $100 \mathrm{~K}$, respectively. Other parameters were assumed as follows: $\lambda_{Q}=0.56 \mathrm{eV}, \hbar \omega_{Q}=1300 \mathrm{~cm}^{-1}$, and $V$ $=3.5 \mathrm{~cm}^{-1}{ }^{53,54}$ Figure 9 shows the theoretical prediction with $\hbar \omega_{H}=250 \mathrm{~cm}^{-1}$ as a high-frequency mode of the CNPUL glassy solid. The temperature dependence of the CS 
rate below $200 \mathrm{~K}$ was well explained by $k_{\mathrm{CS}}\left(\hbar \omega_{H}\right.$ $\left.=250 \mathrm{~cm}^{-1}, \hbar \omega_{Q}=1300 \mathrm{~cm}^{-1}\right)$. With $\hbar \omega_{H}$ smaller than $250 \mathrm{~cm}^{-1}, k_{\mathrm{CS}}\left(\hbar \omega_{H}, \hbar \omega_{Q}\right)$ would decrease monotonously below $200 \mathrm{~K}$. This suggests that the weak temperature dependence of the CS rate at low temperatures originates in nuclear tunneling due to the high-frequency mode in the $\mathrm{CN}$ PUL glassy solid. The high vibrational quantum $\hbar \omega_{H}$ $=250 \mathrm{~cm}^{-1}$ corresponds to a motion with a relaxation time of ca. $0.1 \mathrm{ps}$, which is probably ascribable to librations in polymer glasses. Thus, it was concluded that CS below $200 \mathrm{~K}$ is effectively coupled to the fast motions due to librations with $\hbar \omega_{H}=250 \mathrm{~cm}^{-1}$ leading to weak temperature dependence of the CS rate at low temperatures by nuclear tunneling.

\section{SUMMARY}

Photoinduced CS of Cz-S-A in the CN-PUL glassy solid was studied in a temperature range of 100-400 K. For Cz-S$2 \mathrm{CF}_{3}, k_{\mathrm{CS}}$ increased above $200 \mathrm{~K}$ while it was independent of temperature below $200 \mathrm{~K}$. The increase in $k_{\mathrm{CS}}$ corresponded to the side-chain relaxation of the CN-PUL glassy solid. The dielectric analysis of the side-chain relaxation with the Cole-Cole distribution function revealed a wide distribution in the dielectric relaxation time $\tau$ for the CN-PUL glassy solid. Thus, the temperature dependence of $k_{\mathrm{CS}}$ was theoretically examined considering the wide distribution of $\tau$. As for the increase in $k_{\mathrm{CS}}$ above $200 \mathrm{~K}, k_{\mathrm{CS}}$ was well explained by a thermally nonequilibrium ET formula $\left\langle k_{\mathrm{CS}}(\tau)\right\rangle_{\mathrm{eff}}$ with the effective dielectric constant $\varepsilon_{\text {eff }}$ and the effective dielectric distribution function $\phi(\tau)_{\mathrm{eff}}=\phi(\tau) I(\tau)$. The effective dielectric constant $\varepsilon_{\text {eff }}$ was evaluated with a fluorescent probe of Cz-TP cyclophane. The effective dielectric distribution function $\phi(\tau)_{\text {eff }}$ was a modified distribution function of $\phi(\tau)$, which was weighted by the population of $\mathrm{EtCz}$ in the excited state $I(\tau)$.

As for the weak temperature dependence of $k_{\mathrm{CS}}$ below $200 \mathrm{~K}, k_{\mathrm{CS}}$ was well explained by a two-mode quantum mechanical ET formula with a high-frequency matrix motion $\hbar \omega_{H}=250 \mathrm{~cm}^{-1}$ and a high-frequency intramolecular motion $\hbar \omega_{Q}=1300 \mathrm{~cm}^{-1}$. Both motions are so rapid that ET can be treated under the thermal equilibrium condition. The highfrequency matrix motion was attributed to librational motions of the polar cyano groups in the CN-PUL glassy solid. The wide variations in temperature dependence of $k_{\mathrm{CS}}$ originates from not only the large dielectric intensity owing to the polar cyano group but also the wide distribution in the dielectric relaxation time, which extended into a time domain as fast as the CS event.

\section{ACKNOWLEDGMENT}

We would like to thank Associate Professor Okimichi Yano for dielectric relaxation measurement of CN-PUL.

\footnotetext{
${ }^{1}$ R. A. Marcus, Annu. Rev. Phys. Chem. 15, 155 (1964).

${ }^{2}$ R. A. Marcus and N. Sutin, Biochim. Biophys. Acta 811, 265 (1985).

${ }^{3}$ R. A. Marcus, Angew. Chem., Int. Ed. Engl. 32, 1111 (1993).

${ }^{4}$ R. A. Marcus, J. Electroanal. Chem. 438, 251 (1997).

${ }^{5}$ D. DeVault, Quantum Mechanical Tunneling in Biological Systems (Cambridge University Press, Cambridge, 1984).

${ }^{6}$ G. L. Cross and J. R. Miller, Science 240, 440 (1988).
}

${ }^{7}$ P. F. Barbara, T. J. Meyer, and M. A. Ratner, J. Phys. Chem. 100, 13148 (1996).

${ }^{8}$ A. M. Kuznetsov and J. Ulstrup, Electron Transfer in Chemistry and Biology Wiley, New York, 1999).

${ }^{9}$ T. Asahi, M. Ohkohchi, R. Matsusaka, N. Mataga, R. P. Zhang, A. Osuka, and K. Maruyama, J. Am. Chem. Soc. 115, 5565 (1993).

${ }^{10}$ H. Sumi and R. A. Marcus, J. Chem. Phys. 84, 4894 (1986).

${ }^{11}$ M. J. Weaver and G. E. McManis III, Acc. Chem. Res. 23, 294 (1990).

${ }^{12}$ M. J. Weaver, Chem. Rev. (Washington, D.C.) 92, 463 (1992).

${ }^{13}$ H. Heitele, Angew. Chem., Int. Ed. Engl. 32, 359 (1993).

${ }^{14} \mathrm{~K}$. Yoshihara, K. Tominaga, and Y. Nagasawa, Bull. Chem. Soc. Jpn. 68, 696 (1995).

${ }^{15}$ H. Heitele, M. E. Michel-Beyerle, and P. Finckh, Chem. Phys. Lett. 138, 237 (1987).

${ }^{16}$ P. Finckh, H. Heitele, and M. E. Michel-Beyerle, Chem. Phys. 138, 1 (1989).

${ }^{17}$ A. Masad, D. Huppert, and E. M. Kosower, Chem. Phys. 144, 391 (1990).

${ }^{18}$ U. Rempel, B. von Maltzan, and C. von Borczyskowski, Z. Phys. Chem. 170, 107 (1991).

${ }^{19}$ A. Pugzlys, H. P. den Hartog, A. Baltuska, M. S. Pshenichnikov, S. Umapathy, and D. A. Wiersma, J. Phys. Chem. A 105, 11407 (2001).

${ }^{20}$ T. J. Kang, M. A. Kahlow, D. Giser, S. Swallen, V. Nagarajan, W. Jarzeba, and P. F. Barbara, J. Phys. Chem. 92, 6800 (1988).

${ }^{21}$ K. Tominaga, G. C. Walker, W. Jarzeba, and P. F. Barbara, J. Phys. Chem. 95, 10475 (1991).

${ }^{22}$ G. C. Walker, E. Akesson, A. E. Johnson, N. E. Levinger, and P. F. Barbara, J. Phys. Chem. 96, 3728 (1992).

${ }^{23}$ P. F. Barbara, G. C. Walker, and T. P. Smith, Science 256, 975 (1992).

${ }^{24}$ M. Maroncelli, J. Maclnnis, and G. R. Fleming, Science 243, 1674 (1989).

${ }^{25}$ M. J. Weaver, G. E. McManis, W. Jarzeba, and P. F. Barbara, J. Phys. Chem. 94, 1715 (1990).

${ }^{26}$ J. M. Zaleski, C. K. Chang, G. E. Leroi, R. I. Cukier, and D. G. Nocera, J. Am. Chem. Soc. 114, 3564 (1992).

${ }^{27}$ A. Yartsev, Y. Nagasawa, A. Douhal, and K. Yoshihara, Chem. Phys. Lett. 207, 546 (1993).

${ }^{28}$ O. Nicolet and E. Vauthey, J. Phys. Chem. A 106, 5553 (2002).

${ }^{29}$ Y. Nagasawa, A. P. Yartsev, K. Tominaga, A. E. Johnson, and K. Yoshihara, J. Chem. Phys. 101, 5717 (1994).

${ }^{30}$ Y. Nagasawa, A. P. Yartsev, K. Tominaga, P. B. Bisht, A. E. Johnson, and K. Yoshihara, J. Phys. Chem. 99, 653 (1995).

${ }^{31}$ J. Jortner and M. Bixon, J. Chem. Phys. 88, 167 (1988).

${ }^{32}$ M. Bixon and J. Jortner, Chem. Phys. 176, 467 (1993).

${ }^{33}$ J. T. Hynes, J. Phys. Chem. 90, 3701 (1986).

${ }^{34}$ L. D. Zusman, Chem. Phys. 119, 51 (1988).

${ }^{35}$ G. E. McManis, A. Gochev, and M. Weaver, J. Chem. Phys. 152, 107 (1991).

${ }^{36}$ P. F. Barbara and W. Jarzeba, Adv. Photochem. 15, 1 (1990).

${ }^{37}$ S. J. Rosenthal, X. Xie, M. Du, and G. R. Fleming, J. Chem. Phys. 95, 4715 (1991).

${ }^{38}$ M. Cho, S. J. Rosenthal, N. F. Scherer, L. D. Ziegler, and G. R. Fleming, J. Chem. Phys. 96, 5033 (1992).

${ }^{39}$ R. Jimenez, G. R. Fleming, P. V. Kumar, and M. Maroncelli, Nature (London) 369, 471 (1994).

${ }^{40}$ T. Joo, Y. Jia, and G. R. Fleming, J. Chem. Phys. 102, 4063 (1995).

${ }^{41}$ G. R. Fleming and M. Cho, Annu. Rev. Phys. Chem. 47, 109 (1996).

${ }^{42}$ T. Joo, Y. Jia, J. Y. Yu, M. J. Lang, and G. R. Fleming, J. Chem. Phys. 104, 6089 (1996).

${ }^{43}$ S. A. Passino, Y. Nagasawa, T. Joo, and G. R. Fleming, J. Phys. Chem. A 101, 725 (1997).

${ }^{44}$ L. A. Dick, I. Malfant, D. Kuila, S. Nebolsky, J. M. Nocek, B. M. Hoffman, and M. A. Ratner, J. Am. Chem. Soc. 120, 11401 (1998).

${ }^{45}$ K. V. Mikkeksen and M. A. Ratner, Chem. Rev. (Washington, D.C.) 87, 113 (1987).

${ }^{46}$ P. Chen and T. J. Meyer, Chem. Rev. (Washington, D.C.) 98, 1439 (1998).

${ }^{47}$ H. Fröhlich, Theory of Dielectrics, 2nd ed. (Oxford University Press, London, 1958).

${ }^{48}$ C. J. F. Böttcher and P. Bordewijk, Theory of Electric Polarization, 2nd ed. (Elsevier, Amsterdam, 1978), Vol. II.

${ }^{49}$ F. E. Karasz, Dielectric Properties of Polymers (Plenum, New York, 1972).

${ }^{50}$ N. G. McCrum, B. E. Read, and G. Williams, Anelastic and Dielectric 
Effects in Polymeric Solids (Dover, New York, 1967).

${ }^{51}$ R. T. Bailey, A. M. North, and R. A. Pethrick, Molecular Motion in High Polymers (Oxford University Press, New York, 1981).

${ }^{52}$ A. J. Bur, Polymer 26, 963 (1985).

${ }^{53}$ H. Ohkita, H. Benten, A. Anada, H. Noguchi, N. Kido, S. Ito, and M. Yamamoto, Phys. Chem. Chem. Phys. 6, 3977 (2004)

${ }^{54}$ H. Benten, H. Ohkita, S. Ito, M. Yamamoto, Y. Tohda, and K. Tani, J. Phys. Chem. B 108, 16457 (2004).

${ }^{55}$ P. Lunkenheimer, U. Schneider, R. Brand, and A. Loidl, Contemp. Phys. 41, 15 (2000)

${ }^{56}$ Y. Nagasawa, S. A. Passino, T. Joo, and G. R. Fleming, J. Chem. Phys. 106, 4840 (1997).

${ }^{57}$ Y. Nagasawa, J. Y. Yu, M. Cho, and G. R. Fleming, Faraday Discuss. 108, 23 (1997).

${ }^{58}$ Y. Nagasawa, J. Y. Yu, and G. R. Fleming, J. Chem. Phys. 109, 6175 (1998).

${ }^{59}$ G. R. Fleming, S. A. Passino, and Y. Nagasawa, Philos. Trans. R. Soc. London, Ser. A 356, 389 (1998).

${ }^{60}$ Y. Nagasawa, K. Seike, T. Muromoto, and T. Okada, J. Phys. Chem. A 107, 2431 (2003)

${ }^{61}$ K. S. Cole and R. H. Cole, J. Chem. Phys. 9, 341 (1941).

${ }^{62} \mathrm{H}$. Benten, H. Ohkita, S. Ito, M. Yamamoto, K. Tani, and Y. Tohda, Polymer 45, 2831 (2004).

${ }^{63}$ K. Tani, K. Matsumura, E. Togo et al., Chem. Lett. 32, 910 (2003).

${ }^{64}$ H. Ohkita, S. Ito, M. Yamamoto, K. Tani, and Y. Tohda, J. Phys. Chem. A 106, 2140 (2002).
${ }^{65}$ In the model calculations [Eqs. (10) and (12)], the averaged rates are evaluated by direct averaging of the individual rates, which is a reasonable way for calculating the averaged rates in inhomogeneous media. Therefore, it is true that the averaged experimental rate constant by using the following equation would be more reasonable: $k=\sum_{i=1}^{n} A_{i}\left(1 / \tau_{i}\right.$ $-1 / \tau_{0}$ ), where $n$ represents the number of different relaxation modes in CN-PUL, $A_{i}$ is the relative amplitude of the fluorescence decay component $i$ with lifetime $\tau_{i}$, and $\tau_{0}$ is the fluorescence lifetime of the reference compound. We think, however, that this approach is unrealistic because we cannot decide the number of relaxation modes $n$. Moreover, the number of exponential functions available for fitting experimental data is usually limited to two or three, although it should be larger number $n$. From these reasons, we calculate the experimental CS rate by using the averaged fluorescence lifetime.

${ }^{66}$ One of the three components is ascribable to an impurity $\mathrm{Cz}$ fluorescence that lacks the A moiety, which is a minor component less than 5\%. Thus, $k_{\mathrm{CS}}$ was calculated by $k_{\mathrm{CS}}=1 /\left\langle\tau_{f}\right\rangle-1 / \tau_{f}^{0}$, where $\left\langle\tau_{f}\right\rangle$ is the averaged lifetime of the two major components in the fluorescence of Cz-S-A. See Ref. 53.

${ }^{67}$ J. Jortner, J. Chem. Phys. 64, 4860 (1976).

${ }^{68}$ A. Weller, Z. Phys. Chem., Neue Folge 133, 93 (1982).

${ }^{69}$ A. K. Chibisov, Usp. Khim. 50, 1169 (1981), Russ. Chem. Rev. 50, 615 (1981).

${ }^{70}$ I. Rips and J. Jortner, Chem. Phys. Lett. 133, 411 (1987).

${ }^{71}$ I. Rips and J. Jortner, J. Chem. Phys. 87, 2090 (1987).

${ }^{72}$ H. Benten, H. Ohkita, S. Ito, M. Yamamoto, K. Tani, and Y. Thoda, Bull. Chem. Soc. Jpn. 77, 393 (2004). 